\section{Les vieilles traditions s'écroulent, les temps changent...}

Depuis le numéro 1 de cette année, vous pouvez lire gratuitement l'intégralité des articles du Journal Suisse de Médecine sur Internet. La homepage, créée en 1996, s'est ainsi élargie d'un service remarquable.

Voilà pour le "comment". Il reste à expliquer le "pourquoi". La planète des éditions médicales fait partie de la galaxie Gutenberg. Au premier abord, cela n'est guère compatible avec Internet. Et de plus: qui est assez idiot pour mettre gratuitement sur Internet ce qu'il vend avec succès par ailleurs?

Lorsque, l'année dernière, le directeur $d u \mathrm{Na}$ tional Institut of Health américain, le Dr Harold Varmus, a présenté le concept d'une base de données online comprenant le texte complet des travaux de recherches peer-reviewed de la biomédecine, il a déclenché une vague de réactions, pour la plupart réflexes de défense bien compréhensibles de la part des éditeurs. Les rédactions et les auteurs présentèrent aussi des arguments qualitatifs dans la discussion. Le débat "PubMed Central» est loin d'être clos.

Mais peu d'autres magazines médicaux spécialisés ont osé se jeter à l'eau. En général, les textes complets ne sont disponibles sur Internet que pour les abonnés. Mais pour combien de temps encore estil possible d'en fermer l'accès?

Mettre le contenu des journaux sur Internet et l'ouvrir à tous, de n'importe où et n'importe quand, est certainement la révolution la plus radicale de l'histoire des publications scientifiques depuis la fondation des premiers journaux peer-reviewed, il y a plus de 300 ans. Pour les auteurs, l'accès à leur secteur de recherche quelle que soit leur situation géographique est un énorme avantage. Des archives online complétées d'outils de recherche performants sont aujourd'hui l'une des raisons majeures pour consulter les pages Internet.

Une revue médicale pourvue d'un système peerreview bien conçu est un service essentiel pour les auteurs, qu'il faut perfectionner en permanence. C'est seulement ainsi que la qualité des travaux publiés peut être accrue - et ceci au profit des lecteurs.

"Because Varmus's plan is likely to get bogged down in political and commercial wrangling, we plan to press ahead with an experiment that may work because it has already worked within the high energy physics and astronomy communities" ont déjà remarqué les éditeurs du British Medical Journal en juin 1999 (Br Med J 1999;318:1637-9). Le Canadian Medical Association Journal prophétise: "Medical journals are dead. Long live medical journals" (CMAJ 2000;162:517-8).

Et Gutenberg?

Grâce à son invention, la Bible a pu être imprimée en masse, et tous ceux qui en étaient privés jusqu'alors ont pu lire ce texte fondamental. Internet pourrait jouer le même rôle pour les publications médicales. Les éditeurs médicaux sont des professionnels de la publication. Nous ne devons pas abandonner Internet à des amateurs. C'est pourquoi le Journal Suisse de Médecine est désormais online. Le Bulletin des médecins suisses va bientôt lui faire suite. Tout à fait dans la tradition Gutenberg.

Natalie Marty

EMH Editions médicales suisses SA 